\title{
Self-triggered Min-max DMPC for Asynchronous Multi-agent Systems with Communication Delays
}

\author{
Henglai Wei, Kunwu Zhang, Yang Shi
}

\begin{abstract}
This paper studies the formation stabilization problem of asynchronous nonlinear multi-agent systems (MAS) subject to parametric uncertainties, external disturbances and bounded time-varying communication delays. A self-triggered min-max distributed model predictive control (DMPC) approach is proposed to address this problem. At triggering instants, each agent solves a local min-max optimization problem based on local system states and predicted states of neighbors, determines its next triggering instant and broadcasts its predicted state trajectory to the neighbors. As a result, the communication load is greatly alleviated while retaining robustness and comparable control performance compared to periodic DMPC algorithms. In order to handle time-varying delays, a novel consistency constraint is incorporated into each local optimization problem to restrict the deviation between the newest predicted states and previously broadcast predicted states. Consequently, each agent can utilize previously predicted states of its neighbors to achieve cooperation in the presence of the asynchronous communication and time-varying delays. The proposed algorithm's recursive feasibility and MAS's closed-loop stability at triggering instants are proven. Finally, numerical simulations are conducted to verify the theoretical results.
\end{abstract}

Index Terms-Asynchronous MAS; Min-max DMPC; Distributed self-triggered scheduling; Communication delays.

\section{INTRODUCTION}

Recently, the research on multi-agent systems (MAS) has attracted considerable attention due to its wide range of industrial applications, such as intelligent transportation systems [1], [2], energy systems [3], [4] and multi-robot systems [5]. However, these MAS may suffer from several challenging issues, such as the heavy communication burden, time-varying communication delays, and uncertainties. Hence, it is desirable to develop a robust control algorithm to simultaneously handle these issues of the MAS subject to state and input constraints.

For MAS, the periodic implementation of distributed control algorithms may lead to an undesirable communication burden and induce a nontrivial energy consumption. Alternatively, the event-based distributed control method is a promising solution to reduce the communication load of MAS since the control inputs are only updated and transmitted to actuators at triggering time instants [6]-[9]. In contrast with these methods, event-based distributed MPC (DMPC) has the advantage of systematically handling the constraints of MAS, reducing the communication burden, while optimizing the

This work was supported by the Natural Sciences and Engineering Research Council of Canada (NSERC). (Corresponding author: Yang Shi.)

H. Wei, K. Zhang, Y. Shi are with the Department of Mechanical Engineering and the Institute for the Integrated Energy Systems (IESVic), University of Victoria, Victoria, BC, V8W 3P6, Canada (e-mail: henglaiwei@uvic.ca; kunwu@uvic.ca; yshi@uvic.ca). control performance. Based on different triggering conditions, the triggered DMPC in existing literature can be mainly classified into two categories: event-triggered [10]-[12], and self-triggered DMPC [13], [14]. The authors in [14] present a self-triggered DMPC algorithm for the consensus problem of synchronous linear MAS. However, these communicationefficient DMPC schemes result in the asynchronous information communication among agents, which may degrade the control performance (or even destroy the closed-loop stability) due to the inaccurate information of neighbors [15]. The authors in [13] propose a co-design self-triggered DMPC method for the linear MAS with asynchronous communication. To be specific, the previously broadcast information is used to estimate current optimal states; however, the estimation error induced by the asynchronous communication is not explicitly handled. Some promising results along the research line of the coordination of asynchronous MAS can be found in, for example, [11], [16]. On the other hand, the delay-free transmission among MAS, assumed in the literature mentioned above, is impractical. Understandably, the time-varying communication delays may prevent agents from achieving the control objective. This issue has been studied in [17], [18]. We note that most of these works only concentrate on one of these issues while ignoring others. Simultaneously addressing the aforementioned issues of MAS remains difficult, especially for nonlinear MAS with state and control constraints.

Another challenge is how to guarantee the robustness of the controlled nonlinear MAS when both additive disturbances and parametric uncertainties are presented. Min-max MPC has proven to be an effective method to address this problem [19], [20], where these two types of uncertainties can be tackled by minimizing the cost function related to the worst-case realizations of the uncertainties. To simultaneously overcome the above-mentioned challenges, we propose a self-triggered min-max DMPC for the nonlinear MAS.

The main contribution of this work is threefold.

- A self-triggered min-max DMPC method is proposed for the asynchronous nonlinear MAS subject to parametric uncertainties, external disturbances and bounded timevarying delays. The proposed method can considerably reduce the communication burden and the frequency of solving the optimization problems while attaining the control performance comparable to the periodic DMPC.

- A new consistency constraint that restricts the deviation between the current predicted and previously broadcast states is developed and incorporated into the local optimization problem. Consequently, agents achieve cooperation in the presence of time-varying delays.

- The recursive feasibility of the algorithm is analyzed and 
the sufficient condition on guaranteeing the feasibility is provided. The closed-loop MAS are proven to be inputto-state practically stable (ISpS) at triggering instants.

The remainder of this paper is organized as follows: Section II describes the formation stabilization control problem of nonlinear MAS. In Section III, the optimization problem and the self-triggered scheduler are presented. Section IV provides the theoretical analysis of the recursive feasibility and the closed-loop stability. The simulation study is conducted in Section V] Finally, the conclusion is given in Section VI

The symbols $\mathbb{R}, \mathbb{R}_{\geq 0}, \mathbb{I}$ and $\mathbb{I}_{[m, n]}$ denote the sets of real numbers, nonnegative real numbers, the nonnegative integers and integers in $[m, n], m<n$, respectively. For $x \in \mathbb{R}^{n},\|x\|$ denotes the Euclidean norm, $\|x\|_{P}$ denotes the weighted norm $\sqrt{x^{\mathrm{T}} P x}$, where the matrix $P$ is positive definite. Given two sets $\mathcal{X}, \mathcal{Y} \subseteq \mathbb{R}^{n}$, the set operation $\mathcal{X} \backslash \mathcal{Y}$ is defined by $\mathcal{X} \backslash \mathcal{Y}:=$ $\{x \mid x \in \mathcal{X}, x \notin \mathcal{Y}\} \cdot \bar{\lambda}(P)$ and $\underline{\lambda}(P)$ denote the largest and smallest eigenvalues of the matrix $P$, respectively. $x(t)$ denotes the state $x$ at time $t$ and $x(s \mid t)$ denotes the predicted state at future time $t+s$ determined at time $t, s \in \mathbb{I}$.

\section{PREliminaries AND PROBlem Formulation}

\section{A. Preliminaries}

Consider a discrete-time perturbed nonlinear system

$$
x^{+}=g(x, d)
$$

where $x \in \mathbb{R}^{n}$ is the state, $d=[w, v] \in \mathbb{D} \subset \mathbb{R}^{d}$ is the uncertainty, $x^{+}$is the successor state and $g: \mathbb{R}^{n} \times \mathbb{R}^{d} \rightarrow \mathbb{R}^{n}$. $\mathbb{D}$ is a compact set and contains the origin in its interior. $w$ is the external disturbance and $v$ is the parametric uncertainty.

Before formulating the control problem, we present the following definitions and lemma.

Definition 1: [19] A set $\Omega \subset \mathbb{R}^{n}$ is robustly positive invariant (RPI) for the uncertain system $x^{+}=g(x, d)$, if $g(x, d) \in \Omega$, for all $x \in \Omega$ and all $d \in \mathbb{D}$.

Definition 2: [19] For the system $x^{+}=g(x, u, d)$, a set $\Omega \subset$ $\mathbb{R}^{n}$ is robust control invariant ( $\left.\mathrm{RCI}\right)$, if for all $x \in \Omega$, there exists an admissible control input $u$, such that $g(x, u, d) \in \Omega$, for all $d \in \mathbb{D}$. The $\ell$-step robustly stabilizable set $\mathbb{X}^{\ell}(\Omega)$, i.e., $\forall x \in \mathbb{X}^{\ell}(\Omega)$, the system can be robustly steered into $\Omega$ in $\ell$ steps, is denoted as $\mathbb{X}^{\ell}(\Omega):=\{x \in \mathbb{X} \mid \exists u \in \mathbb{U}$ such that $\left.g(x, u, \mathbb{D}) \subseteq \mathbb{X}^{\ell-1}(\Omega)\right\}$, where $g(x, u, \mathbb{D}):=\{g(x, u, d) \mid d \in$ $\mathbb{D}\}$. In addition, $\mathbb{X}^{0}(\Omega)=\Omega$.

Lemma 1: [19] For the system in (1) with an RPI set $\Pi$, a function $V(\cdot): \mathbb{R}^{n} \rightarrow \mathbb{R}_{\geq 0}$ is called an ISpS Lyapunov function, if it satisfies

$$
\begin{gathered}
\alpha_{1}(\|x\|) \leq V(x) \leq \alpha_{2}(\|x\|)+c_{1}, \\
V\left(x^{+}\right)-V(x) \leq-\alpha_{3}(\|x\|)+\gamma(\|w\|)+c_{2},
\end{gathered}
$$

for all $x \in \Pi, d \in \mathbb{D}$, where constants $c_{1}, c_{2} \in \mathbb{R}_{\geq 0}, \alpha_{1}(\cdot)$, $\alpha_{2}(\cdot), \alpha_{3}(\cdot)$ are $\mathcal{K}_{\infty}$ functions, and $\gamma(\cdot)$ is $\mathcal{K}$ function. If the system admits an ISpS Lyapunov function, then it is ISpS.

Consider the MAS consisting of $M$ dynamically decoupled nonlinear agents. The communication topology among agents is described by a directed graph $\mathcal{G}(\mathcal{M}, \mathcal{E})$ with $\mathcal{E}:=$ $\{(i, j) \mid i, j \in \mathcal{M}, i \neq j\}$ and $\mathcal{M}:=\{1,2, \ldots, M\}$. Let
$\mathcal{N}_{i}=\{j \mid(i, j) \in \mathcal{E}\}$ denote the set of indices of agent $i$ 's neighbors and $n_{i}$ be the cardinality of $\mathcal{N}_{i}$. An edge $(i, j) \in \mathcal{E}$ means that agent $i$ can send message to agent $j$. A sequence of edges $\left(j_{1}, j_{2}\right), \ldots,\left(j_{k-1}, j_{k}\right)$ with $\left(j_{p-1}, j_{p}\right) \in \mathcal{E}$ for all $p \in\{2, \ldots, k\}$ is a directed path from $j_{1}$ to $j_{k}$. The graph $\mathcal{G}(\mathcal{M}, \mathcal{E})$ is assumed to be connected, and each agent can broadcast the information to its neighbors. The discrete-time dynamics of agent $i, i \in \mathcal{M}$ takes the following form

$$
x_{i}(t+1)=f\left(x_{i}(t), u_{i}(t), d_{i}(t)\right), t \in \mathbb{I},
$$

where $x_{i} \in \mathbb{R}^{n}, u_{i} \in \mathbb{R}^{m}$ and $d_{i}=\left[w_{i}, v_{i}\right] \in \mathbb{R}^{d}$ are, respectively, the state, the control input and the uncertainty. Agent $i$ is subject to the state and control input constraints

$$
x_{i}(t) \in \mathbb{X}_{i} \text { and } u_{i}(t) \in \mathbb{U}_{i},
$$

where the state constraint set $\mathbb{X}_{i} \subset \mathbb{R}^{n}$ is closed and the control input set $\mathbb{U}_{i} \subset \mathbb{R}^{m}$ is compact. The disturbance $d_{i}=\left[w_{i}, v_{i}\right]$ is bounded, i.e., $d_{i} \in \mathbb{D}_{i} \subset \mathbb{R}^{d} ; \mathbb{D}_{i}:=\mathbb{W}_{i} \times \mathbb{V}_{i}$, with the external disturbance $w_{i} \in \mathbb{W}_{i} \subset \mathbb{R}^{w}$ and the parametric uncertainty $v_{i} \in \mathbb{V}_{i} \subset \mathbb{R}^{v}$; its upper bound is given by $\bar{d}:=\max _{d_{i} \in \mathbb{D}_{i}}\left\|d_{i}\right\| . \mathbb{W}_{i}$ and $\mathbb{V}_{i}$ are compact and contain the origin in their interiors. The function $f: \mathbb{R}^{n} \times \mathbb{R}^{m} \times \mathbb{R}^{d} \rightarrow \mathbb{R}^{n}$ is assumed to be differentiable and satisfies $f(0,0,0)=0$.

Assumption 1: There exist constants $\nu, \xi \in \mathbb{R}_{\geq 0}$ such that the conditions $\|f(x, u, d)-f(y, u, d)\| \leq \nu\|x-y\|$ and $\left\|f(x, u, d)-f\left(x, u, d^{\prime}\right)\right\| \leq \xi\left\|d-d^{\prime}\right\|$ hold for all $x, y \in \mathbb{X}_{i}$, $u \in \mathbb{U}_{i}, d, d^{\prime} \in \mathbb{D}_{i}, i \in \mathcal{M}$.

Assumption 1 helps to quantify an upper bound on the deviation between the newest and assumed predicted state. $\nu$ and $\xi$ can be calculated following [22] (see Lemmas 3.2, 3.3).

Let $t_{k}^{i}, k \in \mathbb{I}$ be the triggering instant of agent $i, i \in \mathcal{M}$. Agent $i$ measures the local system state, receives neighbors' predicted state information, and applies the calculated control action at $t_{k}^{i}$. The sampling instants can be denoted as a sequence $\left\{t_{k}^{i}\right\}, k \in \mathbb{I}$.

Definition 3: [23] The MAS in (2) are synchronous if for $i, j \in \mathcal{M},\left\{t_{k}^{i}\right\}=\left\{t_{k}^{j}\right\}$, i.e., $t_{k}^{i}=t_{k}^{j}, \forall k \in \mathbb{I}$. The MAS are said to be asynchronous if for $i, j \in \mathcal{M}, i \neq j,\left\{t_{k}^{i}\right\}$ is independent of $\left\{t_{k}^{j}\right\}$, i.e, agents may not update their system states at the same time.

The local sampling time $t_{k}^{i}, i \in \mathcal{M}$ and the communication delays $\tau_{k}^{i j}$ (from agent $j$ to $i$ ) satisfy the following assumption. If the sampling period and communication delays are arbitrarily large, it becomes difficult to guarantee the closedloop stability property of the MAS without new measurements. Thus, the following assumption is given.

Assumption 2: For agent $i, i \in \mathcal{M}$, the local sampling instant $t_{k}^{i}$ and the communication delays $\tau_{k}^{i j}, k \in \mathbb{I}$, satisfy: 1) $1 \leq t_{k+1}^{i}-t_{k}^{i} \leq \bar{H}$; 2) $0<\tau_{k}^{i j} \leq \bar{\tau}$; 3) There is no disordering transmission among agents. Here the largest admissible sampling interval $\bar{H} \in \mathbb{I}$ and the largest communication delay $\bar{\tau} \in \mathbb{I}$ are finite.

\section{B. Problem formulation}

The objective is to design a robust DMPC method for nonlinear MAS with time-varying delays, parametric uncertainties, and external disturbances, such that the MAS are robustly 


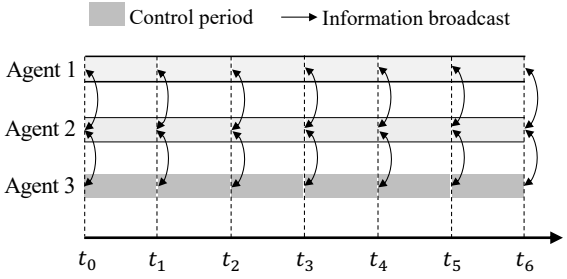

(a) Periodic and synchronous 21

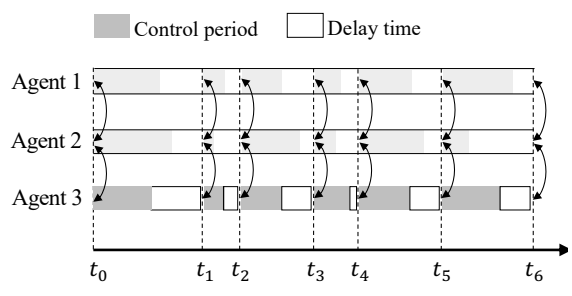

(b) Aperiodic and synchronous

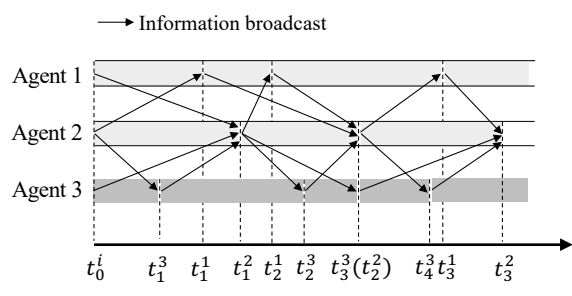

(c) Aperiodic and asynchronous

Fig. 1: An example of MAS which consists of three agents: $\mathcal{N}_{1}=\{2\}, \mathcal{N}_{2}=\{1,3\}, \mathcal{N}_{3}=\{2\}$. Periodic versus aperiodic and synchronous versus asynchronous DMPC methods are illustrated. For the synchronous DMPC method, all agents simultaneously update the states at time $t_{k}, k \in \mathbb{I}$. In (b), the update time $t_{k}, k \in \mathbb{I}$ is aperiodically synchronized based on the maximum communication delay. In (c), each agent asynchronously updates and transmits the system state without waiting for its neighbors.

stabilized. Furthermore, a distributed self-triggered scheduler is developed to reduce the communication burden and the frequency of solving the corresponding optimization problems.

\section{Asynchronous communication with time-varying delays}

At $t_{k}^{i}$, agent $i, i \in \mathcal{M}$ broadcasts the newest predicted state sequence $\boldsymbol{x}_{i}^{b}\left(t_{k}^{i}\right):=\left(x_{i}^{b}\left(\cdot \mid t_{k}^{i}\right)\right)$ to its neighbors as shown in Fig. 1(c), which is constructed as

$$
x_{i}^{b}\left(s \mid t_{k}^{i}\right)= \begin{cases}x_{i}^{*}\left(s \mid t_{k}^{i}\right), & s \in \mathbb{I}_{[0, N)} \\ 0, & s \in \mathbb{I}_{\left[N, H^{i *}\left(t_{k}^{i}\right)+\bar{\tau}+N\right]},\end{cases}
$$

where $x_{i}^{*}\left(s \mid t_{k}^{i}\right)$ denotes the optimal predicted state and $H^{i *}\left(t_{k}^{i}\right) \in \mathbb{I}_{[1, \bar{H}]}$ is the optimal triggering interval at $t_{k}^{i}$. The calculation of $x_{i}^{*}\left(s \mid t_{k}^{i}\right)$ and $H^{i *}\left(t_{k}^{i}\right)$ will be introduced in Section III

Now we describe the bounded time-varying communication delays for the asynchronous MAS. Let $\boldsymbol{x}_{j}^{b}\left(t_{k}^{i j}\right)$ represent the newest message broadcast by agent $j, j \in \mathcal{N}_{i}$ at $t_{k}^{i j}$, where $t_{k}^{i j}:=\max \left\{t_{l}^{j} \in \mathbb{I} \mid t_{l}^{j}<t_{k}^{i}\right\}$. The communication delays can be categorized as the following two cases:

Case 1: $0 \leq \tau_{k}^{i j} \leq t_{k}^{i}-t_{k}^{i j}$, i.e., the newest message $\boldsymbol{x}_{j}^{b}\left(t_{k}^{i j}\right)$ of agent $j$ is received by agent $i$ at $t_{k}^{i}$;

Case 2: $t_{k}^{i}-t_{k}^{i j}<\tau_{k}^{i j} \leq \bar{\tau}$, i.e., the newest message $\boldsymbol{x}_{j}^{b}\left(t_{k}^{i j}\right)$ cannot be received by agent $i$ at $t_{k}^{i}$, therefore, agent $i$ can only utilize the message $\boldsymbol{x}_{j}^{b}\left(t_{k-1}^{i j}\right)$ broadcast at previous time instants, which includes the neighbors' state information $x_{j}^{b}\left(s \mid t_{k-1}^{i j}\right), s \in \mathbb{I}_{\left[0, H^{i *}\left(t_{k-1}^{i j}\right)+\bar{\tau}+N\right]}$.

Consequently, the predicted state trajectories of neighbors $\boldsymbol{x}_{j}\left(t_{k}^{i}\right), j \in \mathcal{N}_{i}$ used in the local optimization problem of agent $i$ in the next section can be constructed as

$$
\boldsymbol{x}_{j}\left(t_{k}^{i}\right):=\left(x_{j}^{b}\left(0 \mid t_{k}^{i}\right), x_{j}^{b}\left(1 \mid t_{k}^{i}\right), \cdots, x_{j}^{b}\left(N-1 \mid t_{k}^{i}\right)\right) .
$$

At $t_{k}^{i}$, each agent receives its neighbors' newest predicted state sequence $x_{j}^{b}\left(\cdot \mid t_{k}^{j}\right), j \in \mathcal{N}_{i}$ as in (3) and (4) to formulate the objective function for the DMPC optimization problem

$$
J_{i, N}^{H^{i}}\left[t_{k}^{i}\right]=\sum_{s=0}^{H^{i}-1} \frac{1}{\hbar_{i}} L_{i}\left[s \mid t_{k}^{i}\right]+\sum_{s=H^{i}}^{N-1} L_{i}\left[s \mid t_{k}^{i}\right]+F_{i}\left[N \mid t_{k}^{i}\right],
$$

where $J_{i, N}^{H^{i}}\left[t_{k}^{i}\right]=J_{i, N}^{H^{i}}\left(x_{i}\left(t_{k}^{i}\right), \boldsymbol{x}_{-i}\left(t_{k}^{i}\right), \boldsymbol{u}_{i}\left(t_{k}^{i}\right), \boldsymbol{d}_{i}\left(t_{k}^{i}\right)\right)$, $L_{i}\left[s \mid t_{k}^{i}\right]=L_{i}\left(x_{i}\left(s \mid t_{k}^{i}\right), x_{-i}\left(s \mid t_{k}^{i}\right), u_{i}\left(s \mid t_{k}^{i}\right), d_{i}\left(s \mid t_{k}^{i}\right)\right)$,
$F_{i}\left[N \mid t_{k}^{i}\right]=F_{i}\left(x_{i}\left(N \mid t_{k}^{i}\right)\right), N$ is the prediction horizon and $H^{i}\left(t_{k}^{i}\right)$ denotes the triggering interval. The parameter $\hbar_{i}>1$ allows a trade-off between the control performance (with respect to the optimal value of the objective function) and the communication load (i.e., the communication frequency of each agent). $\boldsymbol{x}_{-i}\left(t_{k}^{i}\right):=\left(\boldsymbol{x}_{i_{1}}\left(t_{k}^{i}\right), \ldots, \boldsymbol{x}_{i_{n_{i}}}\left(t_{k}^{i}\right)\right)$ denote the collection of the predicted state sequence of agent $i$ 's neighbors as in (3), with $i_{q} \in \mathcal{N}_{i}$, $q \in \mathbb{I}_{\left[1, n_{i}\right]}, \quad x_{-i}\left(s \mid t_{k}^{i}\right):=\left(x_{i_{1}}^{b}\left(s \mid t_{k}^{i}\right), \ldots, x_{i_{n_{i}}}^{b}\left(s \mid t_{k}^{i}\right)\right)$, $s \in \mathbb{I}_{[0, N]}, \boldsymbol{u}_{i}\left(t_{k}^{i}\right):=\left(u_{i}\left(0 \mid t_{k}^{i}\right), \ldots, u_{i}\left(N-1 \mid t_{k}^{i}\right)\right)$ and $\boldsymbol{d}_{i}\left(t_{k}^{i}\right):=\left(d_{i}\left(0 \mid t_{k}^{i}\right), \ldots, d_{i}\left(N-1 \mid t_{k}^{i}\right)\right)$. The local stage cost of agent $i, i \in \mathcal{M}$ is designed as

$$
\begin{aligned}
L_{i}\left[s \mid t_{k}^{i}\right]= & \left\|x_{i}\left(s \mid t_{k}^{i}\right)\right\|_{Q_{i}}^{2}+\left\|u_{i}\left(s \mid t_{k}^{i}\right)\right\|_{R_{i}}^{2} \\
& +\sum_{j \in \mathcal{N}_{i}}\left\|x_{i}\left(s \mid t_{k}^{i}\right)-x_{j}^{b}\left(s \mid t_{k}^{i}\right)\right\|_{Q_{i j}}^{2}
\end{aligned}
$$

where the weighting matrices $Q_{i}$ and $Q_{i j}$ are symmetric and positive semidefinite, $R_{i}$ is symmetric and positive definite. Intuitively, the coupling cost term $\left\|x_{i}\left(s \mid t_{k}^{i}\right)-x_{j}\left(s \mid t_{k}^{i}\right)\right\|_{Q_{i j}}^{2}$, $j \in \mathcal{N}_{i}$ makes agents to achieve cooperation. The local terminal cost is designed as $F_{i}\left(x_{i}\left(N \mid t_{k}^{i}\right)\right)=\left\|x_{i}\left(N \mid t_{k}^{i}\right)\right\|_{P_{i}}^{2}$, where $P_{i}$ is symmetric and positive semidefinite.

Assumption 3: For agent $i, i \in \mathcal{M}$, the local decoupled terminal set $\Omega_{i} \subseteq \mathbb{X}_{i}$ is an RPI set with a local feedback controller $\kappa_{i}\left(x_{i}(t)\right) \in \mathbb{U}_{i}, t \in \mathbb{I}$. There exist a $\mathcal{K}$ function $\sigma_{i}(\cdot)$ and a constant $c_{4} \in \mathbb{R}_{>0}$ such that $F_{i}\left(x_{i}(t+1)\right)-$ $F_{i}\left(x_{i}(t)\right) \leq-L_{i}\left(x_{i}(t), x_{-i}(t), \kappa_{i}\left(x_{i}(t)\right), d_{i}(t)\right)+\epsilon_{i}$, where $\epsilon_{i}=\sigma_{i}\left(\left\|w_{i}(t)\right\|\right)+c_{4}, x_{-i}(t)=0$, for all $x_{i}(t) \in \Omega_{i}$ and $d_{i}(t) \in \mathbb{D}_{i}$.

When $x_{i}\left(N \mid t_{k}^{i}\right) \in \Omega_{i}$, from the definition of the broadcast state trajectory $x_{i}^{b}\left(\cdot \mid t_{k}^{i}\right), i \in \mathcal{M}$ as in (3), it is easy to know that the state of its neighbors $x_{j}\left(N \mid t_{k}^{i}\right)=x_{j}\left(N+t_{k}^{i}-t_{k}^{j} \mid t_{k}^{j}\right)=0$, $j \in \mathcal{N}_{i}$. Then, the conventional design of the terminal region and terminal controller for the single system (see, e.g., [19], [24]) is also applicable to the MAS.

\section{Self-Triggered Min-Max DMPC}

This section presents a solution to the problem formulated in the previous section based on the min-max DMPC. Furthermore, a distributed self-triggered scheduler for the nonlinear asynchronous MAS with bounded time-varying communication delays is designed. 


\section{A. Min-max DMPC optimization problem}

The min-max optimization problem $\mathcal{P}_{i}$ is designed as

$$
\begin{aligned}
& \min _{u_{i}\left(s \mid t_{k}^{i}\right)}\left\{\max _{d_{i}\left(s \mid t_{k}^{i}\right) \in \mathbb{D}_{i}}\left\{\bar{J}_{i, N}^{H^{i}}\left(x_{i}\left(t_{k}^{i}\right), \boldsymbol{x}_{-i}\left(t_{k}^{i}\right), u_{i}\left(s \mid t_{k}^{i}\right), d_{i}\left(s \mid t_{k}^{i}\right)\right)\right\},\right. \\
&\text { such that } \left.x_{i}\left(H^{i} \mid t_{k}^{i}\right) \in \mathbb{X}_{i}^{N-H^{i}}\left(\Omega_{i}\right), \forall d_{i}\left(s \mid t_{k}^{i}\right) \in \mathbb{D}_{i}\right\} \\
& \text { s.t. } x_{i}\left(s+1 \mid t_{k}^{i}\right)=f\left(x_{i}\left(s \mid t_{k}^{i}\right), u_{i}\left(s \mid t_{k}^{i}\right), d_{i}\left(s \mid t_{k}^{i}\right)\right), \\
& x_{i}\left(0 \mid t_{k}^{i}\right)=x_{i}\left(t_{k}^{i}\right), \\
& u_{i}\left(s \mid t_{k}^{i}\right) \in \mathbb{U}_{i}, \\
& x_{i}\left(s \mid t_{k}^{i}\right) \in \mathbb{X}_{i}, \\
&\left\|x_{i}\left(s \mid t_{k}^{i}\right)-x_{i}^{b}\left(s+t_{k}^{i}-t_{k-1}^{i} \mid t_{k-1}^{i}\right)\right\| \leq \Delta_{i},
\end{aligned}
$$

where the triggering interval $H^{i}\left(t_{k}^{i}\right)$ is abbreviated as $H^{i}$, $s \in \mathbb{I}_{\left[0, H^{i}\right)}, \Omega_{i}:=\left\{x_{i} \in \mathbb{X}_{i} \mid\left\|x_{i}\right\|_{P_{i}} \leq \rho_{i}, \rho_{i} \in \mathbb{R}_{\geq 0}\right\}$ is the terminal set, $\Delta_{i} \in \mathbb{R}_{\geq 0}$ is a design parameter and $V_{i, N}^{H^{i}}\left(x_{i}\left(t_{k}^{i}\right), \boldsymbol{x}_{-i}\left(t_{k}^{i}\right)\right)$ is the optimal value of the objective function of $\mathcal{P}_{i} . \bar{J}_{i, N}^{H^{i}}\left(x_{i}\left(t_{k}^{i}\right), \boldsymbol{x}_{-i}\left(t_{k}^{i}\right), u_{i}\left(s \mid t_{k}^{i}\right), d_{i}\left(s \mid t_{k}^{i}\right)\right):=$ $\sum_{s=0}^{H^{i}-1} \frac{1}{\hbar_{i}} L_{i}\left(x_{i}\left(t_{k}^{i}\right), x_{-i}\left(s \mid t_{k}^{i}\right), u_{i}\left(s \mid t_{k}^{i}\right), d_{i}\left(s \mid t_{k}^{i}\right)\right)$ $V_{i, N-H^{i}}\left(x_{i}\left(H^{i} \mid t_{k}^{i}\right), x_{-i}\left(H^{i} \mid t_{k}^{i}\right)\right)$ with

$$
\begin{aligned}
& V_{i, \ell}\left(x_{i}\left(s \mid t_{k}^{i}\right), x_{-i}\left(s \mid t_{k}^{i}\right)\right) \\
= & \min _{\mu_{i, \ell}\left(s \mid t_{k}^{i}\right) \in \mathbb{U}_{i}}\left\{\operatorname { m a x } _ { d _ { i } ( s | t _ { k } ^ { i } ) \in \mathbb { D } _ { i } } \left\{L _ { i } \left(x_{i}\left(s \mid t_{k}^{i}\right), x_{-i}\left(s \mid t_{k}^{i}\right),\right.\right.\right. \\
& \left.\mu_{i, \ell}\left(s \mid t_{k}^{i}\right), d_{i}\left(s \mid t_{k}^{i}\right)\right)+V_{i, \ell-1}\left(f \left(x_{i}\left(s \mid t_{k}^{i}\right), \mu_{i, \ell}\left(s \mid t_{k}^{i}\right),\right.\right. \\
& \left.\left.\left.d_{i}\left(s \mid t_{k}^{i}\right)\right), x_{-i}\left(s+1 \mid t_{k}^{i}\right)\right)\right\}, \text { such that } \\
& x_{i}\left(s \mid t_{k}^{i}\right) \in \mathbb{X}_{i}^{\ell}\left(\Omega_{i}\right), \\
& f\left(x_{i}\left(s \mid t_{k}^{i}\right), \mu_{i, \ell}\left(s \mid t_{k}^{i}\right), d_{i}\left(s \mid t_{k}^{i}\right)\right) \subseteq \mathbb{X}_{i}^{\ell-1}\left(\Omega_{i}\right) \\
& \left.\left\|x_{i}\left(s \mid t_{k}^{i}\right)-x_{i}^{b}\left(s+t_{k}^{i}-t_{k-1}^{i} \mid t_{k-1}^{i}\right)\right\| \leq \Delta_{i}\right\},
\end{aligned}
$$

where $\mathbb{X}_{i}^{\ell}\left(\Omega_{i}\right)$ is the $\ell$-step robustly stabilizable set $[20]$ of agent $i, \ell=N-s ; V_{i, 0}\left(x_{i}\left(N \mid t_{k}^{i}\right)\right)=F_{i}\left(x_{i}\left(N \mid t_{k}^{i}\right)\right)$. Solving Problem $\mathcal{P}_{i}$ yields the optimal control sequence $\boldsymbol{u}_{i}^{*}\left(t_{k}^{i}\right):=$ $\left(u_{i}^{*}\left(0 \mid t_{k}^{i}\right), \ldots, u_{i}^{*}\left(H^{i}-1 \mid t_{k}^{i}\right), \mu_{i, \ell}^{*}\left(s \mid t_{k}^{i}\right), \ldots, \mu_{i, 1}^{*}\left(N-1 \mid t_{k}^{i}\right)\right)$, where $u_{i}^{*}\left(s \mid t_{k}^{i}\right), s \in \mathbb{I}_{\left[0, H^{i}\right)}$ is the optimal open-loop control action and $\mu_{i, \ell}^{*}\left(s \mid t_{k}^{i}\right), s \in \mathbb{I}_{\left[H^{i}, N\right)}$ is the optimal feedback control law generated by solving (6) via dynamic programming. And $d_{i}^{*}\left(s \mid t_{k}^{i}\right), s \in \mathbb{I}_{\left[H^{i}, N\right)}$ is the optimal disturbance sequence.

Remark 1: Both the communication load and control performance are considered in the objective function of the DMPC optimization problem $\mathcal{P}_{i}$ when $\hbar_{i}>1$. In this context, the increasing value of $\hbar$ weakens the effect of the openloop prediction (i.e., decreasing the weight of the open-loop cost function concerning the overall cost function), while guaranteeing comparable control performance compared to periodic min-max DMPC. A similar design of the parameter $\hbar_{i}$ was exploited for the single system in [24], [25]. Especially, when $H^{i}\left(t_{k}^{i}\right)=1$, the triggered scheme becomes a standard periodic triggered scheme.

Remark 2: The consistency constraint in 5b that specifies an upper bound of the deviation between the newest predicted states and previously broadcast states is incorporated into the local DMPC optimization problem. If the assumed state and the optimal predicted state calculated at the last time instant coincide with each other, the constraint $(5 \mathrm{~b})$ can be reduced to the compatibility constraint in [26] for the synchronous MAS with delay-free and periodic communication. Hence, the proposed consistency constraint is more general and captures the compatibility constraint in [26] as a special case.

\section{B. Distributed self-triggered scheduler}

A distributed self-triggered scheduler is designed to generate a sequence $\left\{t_{k}^{i}\right\}$, based on the local system state and newest neighbors' predicted states, i.e., $t_{k+1}^{i}=t_{k}^{i}+H^{i *}\left(t_{k}^{i}\right)$ and

$$
H^{i *}\left(t_{k}^{i}\right)=\max \left\{H^{i} \in \mathbb{I}_{[1, \bar{H}]} \mid V_{i, N}^{H^{i}}\left[t_{k}^{i}\right] \leq V_{i, N}^{1}\left[t_{k}^{i}\right]\right\},
$$

where $H^{i *}\left(t_{k}^{i}\right)$ is the optimal triggering interval at $t_{k}^{i}$ and $V_{i, N}^{H^{i}}\left[t_{k}^{i}\right]=V_{i, N}^{H^{i}}\left(x_{i}\left(t_{k}^{i}\right), \boldsymbol{x}_{-i}\left(t_{k}^{i}\right)\right) . H^{i *}\left(t_{k}^{i}\right)$ is abbreviated as $H^{i *}$ hereafter. Applying the first $H^{i *}$ open-loop control inputs $\boldsymbol{u}_{i}^{\mathrm{mpc}}\left(t_{k}^{i}\right):=u_{i}^{*}\left(s \mid t_{k}^{i}\right), s \in \mathbb{I}_{\left[0, H^{i *}\right.}$ to the system (2) yields the following closed-loop system

$$
x_{i}\left(t_{k}^{i}+s+1\right)=f\left(x_{i}\left(t_{k}^{i}+s\right), u_{i}^{*}\left(s \mid t_{k}^{i}\right), d_{i}\left(t_{k}^{i}+s\right)\right),
$$

and $d_{i}\left(t_{k}^{i}+s\right), s \in \mathbb{I}_{\left[0, H^{i *}\right)}$ is the actual disturbance sequence.

Remark 3: It is worth mentioning that the existing results on self-triggered DMPC in [13], [14] are restricted to the special case of deterministic linear MAS with perfect communication. In contrast, the proposed triggered mechanism is extended to more general situations, i.e., the nonlinear MAS with two types of uncertainties and communication delays.

\section{Self-triggered asynchronous min-max DMPC algorithm}

The proposed self-triggered DMPC method is summarized in Algorithm 1.

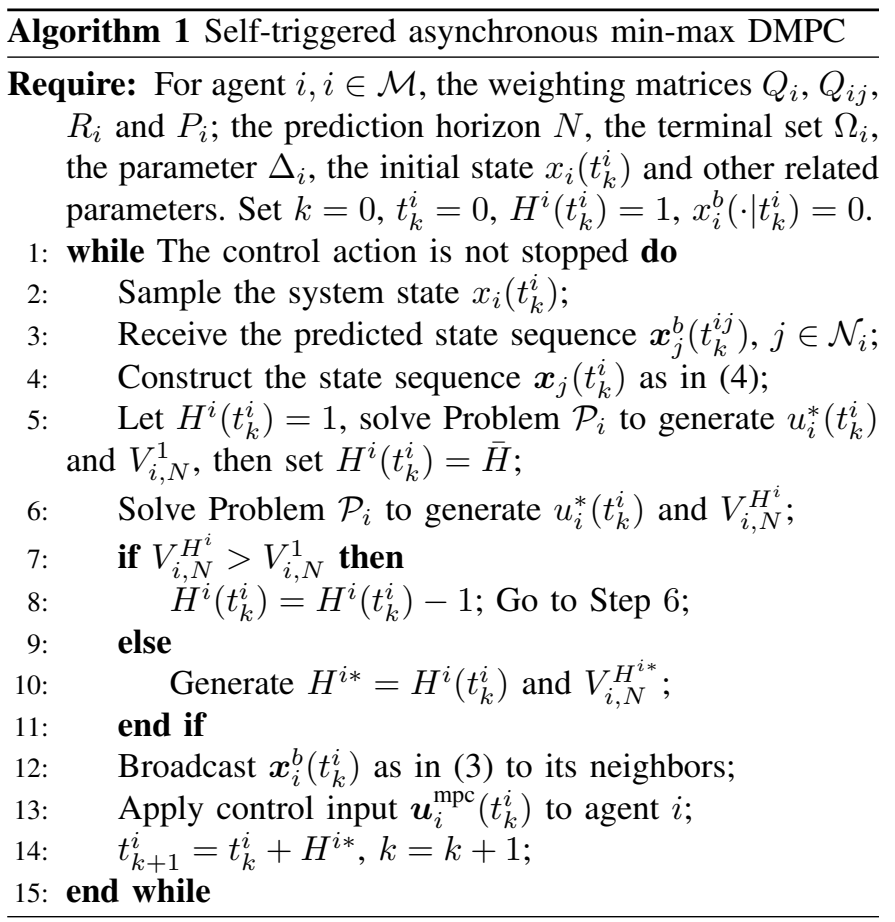

Note that the distributed optimization problem $\mathcal{P}_{i}$ is solved without considering the constraint (5d) in the initialization step. Besides, for each agent, the predicted states of neighbors are assumed to be zeros over the period $\left[t_{0}^{i}, t_{0}^{i}+N\right]$, and the communication delay at $t_{0}^{i}$ is zero, i.e., $\tau_{0}^{i j}=0$. 


\section{THEORETICAL ANALYSIS}

Lemma 2: Under Assumptions 1 and 2, if $\mathcal{P}_{i}$ is feasible at $t_{k}^{i}$, and the condition $\Delta_{i} \geq \max \left\{\nu^{N-1-\bar{H}} \bar{\phi}, \rho_{i} / \underline{\lambda}\left(P_{i}^{1 / 2}\right)\right\}$ holds, where $\bar{\phi}:=2 \sum_{s=0}^{\bar{H}+t_{k}^{i}-t_{\tau}^{i}} \nu^{s} \xi \bar{d}, t_{\tau}^{i} \in\left[t_{k}^{i}-\bar{H}, t_{k}^{i}\right]$, $t_{k+1}^{i}=t_{k}^{i}+H^{i *}$, then, the inequality $\| \tilde{x}_{i}\left(s \mid t_{k+1}^{i}\right)-x_{i}^{b}\left(t_{k+1}^{i}+\right.$ $\left.s-t_{k}^{i} \mid t_{k}^{i}\right) \| \leq \Delta_{i}, s \in \mathbb{I}_{[0, N]}$ holds.

Proof: If $s \in \mathbb{I}_{\left[N-H^{i *}, N\right]}$, it follows from (3) that $x_{i}^{b}\left(t_{k+1}^{i}+s-t_{\tau}^{i} \mid t_{\tau}^{i}\right)=0$, we have $\| \tilde{x}_{i}\left(s \mid t_{k+1}^{i}\right)-x_{i}^{b}\left(t_{k+1}^{i}+\right.$ $\left.s-t_{\tau}^{i} \mid t_{\tau}^{i}\right)\|=\| \tilde{x}_{i}\left(s \mid t_{k+1}^{i}\right) \|$. By the condition $\Delta_{i} \geq$ $\max \left\{\nu^{N-1-\bar{H}} \bar{\phi}, \rho_{i} / \underline{\lambda}\left(P_{i}^{1 / 2}\right)\right\}$, it follows that $\left\|\tilde{x}_{i}\left(s \mid t_{k+1}^{i}\right)\right\| \leq$ $\rho_{i} / \underline{\lambda}\left(P_{i}^{1 / 2}\right) \leq \Delta_{i}$.

If $s=0$, by Assumption 1 , we get

$$
\begin{aligned}
& \left\|\tilde{x}_{i}\left(0 \mid t_{k+1}^{i}\right)-x_{i}^{b}\left(t_{k+1}^{i}-t_{\tau}^{i} \mid t_{\tau}^{i}\right)\right\| \\
\leq & \nu\left\|x_{i}\left(t_{k+1}^{i}-1\right)-x_{i}^{*}\left(t_{k+1}^{i}-1-t_{\tau}^{i} \mid t_{\tau}^{i}\right)\right\| \\
& +\xi\left\|d_{i}\left(t_{k+1}^{i}-1\right)-d_{i}^{*}\left(t_{k+1}^{i}-1-t_{\tau}^{i} \mid t_{\tau}^{i}\right)\right\| \\
\leq & \nu\left\|x_{i}\left(t_{k+1}^{i}-1\right)-x_{i}^{*}\left(t_{k+1}^{i}-1-t_{\tau}^{i} \mid t_{\tau}^{i}\right)\right\|+2 \xi \bar{d} .
\end{aligned}
$$

Since $x_{i}\left(t_{\tau}^{i}\right)=x_{i}^{b}\left(0 \mid t_{\tau}^{i}\right)$, then we can obtain

$$
\left\|\tilde{x}_{i}\left(0 \mid t_{k+1}^{i}\right)-x_{i}^{b}\left(t_{k+1}^{i}-t_{\tau}^{i} \mid t_{\tau}^{i}\right)\right\| \leq 2 \sum_{s=0}^{t_{k+1}^{i}-t_{\tau}^{i}} \nu^{s} \xi \bar{d}=\phi .
$$

Next, we consider $s \in \mathbb{I}_{\left(0, N-H^{i *}\right)}$

$$
\begin{aligned}
& \left\|\tilde{x}_{i}\left(s \mid t_{k+1}^{i}\right)-x_{i}^{b}\left(t_{k+1}^{i}+s-t_{\tau}^{i} \mid t_{\tau}^{i}\right)\right\| \\
= & \| f\left(\tilde{x}_{i}\left(s-1 \mid t_{k+1}^{i}\right), u_{i}^{*}\left(t_{k+1}^{i}+s-1-t_{\tau}^{i} \mid t_{\tau}^{i}\right),\right. \\
& \left.d_{i}^{*}\left(t_{k+1}^{i}+s-1-t_{\tau}^{i} \mid t_{\tau}^{i}\right)\right) \\
& -f\left(x_{i}^{*}\left(t_{k+1}^{i}-1-t_{\tau}^{i} \mid t_{\tau}^{i}\right), u_{i}^{*}\left(t_{k+1}^{i}-1-t_{\tau}^{i} \mid t_{\tau}^{i}\right),\right. \\
& \left.d_{i}^{*}\left(t_{k+1}^{i}-1-t_{\tau}^{i} \mid t_{\tau}^{i}\right)\right) \| \\
\leq & \nu\left\|\tilde{x}_{i}\left(s-1 \mid t_{k+1}^{i}\right)-x_{i}^{*}\left(t_{k+1}^{i}+s-1-t_{\tau}^{i} \mid t_{\tau}^{i}\right)\right\|,
\end{aligned}
$$

which implies

$$
\begin{aligned}
& \| \tilde{x}_{i}\left(N-H^{i *}-1 \mid t_{k+1}^{i}\right) \\
& -x_{i}^{b}\left(t_{k+1}^{i}+N-H^{i *}-1-t_{\tau}^{i} \mid t_{\tau}^{i}\right) \| \\
\leq & \nu^{N-1-H^{i *}}\left\|\tilde{x}_{i}\left(0 \mid t_{k+1}^{i}\right)-x_{i}^{b}\left(t_{k+1}^{i}-t_{\tau}^{i} \mid t_{\tau}^{i}\right)\right\| \\
\leq & \nu^{N-1-H^{i *}} \phi \\
\leq & \nu^{N-1-\bar{H}} \bar{\phi} .
\end{aligned}
$$

Then based on the condition $\Delta_{i} \geq \max \left\{\nu^{N-1-\bar{H}} \bar{\phi}\right.$, $\left.\rho_{i} / \underline{\lambda}\left(P_{i}^{1 / 2}\right)\right\}$, we have

$$
\left\|\tilde{x}_{i}\left(s \mid t_{k+1}^{i}\right)-x_{i}^{b}\left(t_{k+1}^{i}+s-t_{\tau}^{i} \mid t_{\tau}^{i}\right)\right\| \leq \Delta_{i},
$$

which implies that the inequality $\| \tilde{x}_{i}\left(s \mid t_{k+1}^{i}\right)-x_{i}^{b}\left(t_{k+1}^{i}+s-\right.$ $\left.t_{k}^{i} \mid t_{k}^{i}\right) \| \leq \Delta_{i}$ holds, for all $s \in \mathbb{I}_{[0, N]}$.

Lemma 3: Consider the system in (2) and suppose that Assumption 3 holds. Then, $V_{i, \ell+1}\left(x_{i}(t), x_{-i}(t)\right)-$ $V_{i, \ell}\left(x_{i}(t), x_{-i}(t)\right) \leq \max _{d_{i}}\left\{V_{i, \ell}\left(x_{i}(t+1), x_{-i}(t+1)\right)-\right.$ $\left.V_{i, \ell-1}\left(x_{i}(t+1), x_{-i}(t+1)\right)\right\}$, where $t \in \mathbb{I}, \forall x_{i}(t) \in$ $\mathbb{X}_{i}^{\ell}\left(\Omega_{i}\right), \forall x_{j}(t) \in \mathbb{X}_{j}^{\ell}\left(\Omega_{j}\right), j \in \mathcal{N}_{i}, \forall d_{i}(t) \in \mathbb{D}_{i}$. Also, $V_{i, \ell}\left(x_{i}(t), x_{-i}(t)\right)-V_{i, \ell-1}\left(x_{i}(t), x_{-i}(t)\right) \leq \epsilon_{i}$ and $V_{i, \ell}\left(x_{i}(t), x_{-i}(t)\right) \leq V_{i, 0}\left(x_{i}(t)\right)+\ell \epsilon_{i}$.

Proof: The proof can follow Theorem 2 in [19].

Lemma 4: For the constrained min-max DMPC optimization problem $\mathcal{P}_{i}$ in (5), the inequality $V_{i, N}^{1}\left(x_{i}\left(t_{k}^{i}\right), \boldsymbol{x}_{-i}\right.$ $\left.\left(t_{k}^{i}\right)\right) \leq V_{i, N}\left(x_{i}\left(t_{k}^{i}\right), \boldsymbol{x}_{-i}\left(t_{k}^{i}\right)\right)$ holds, where $V_{i, N}\left(x_{i}\left(t_{k}^{i}\right), \boldsymbol{x}_{-i}\right.$ $\left.\left(t_{k}^{i}\right)\right):=J_{i, N}\left(x_{i}\left(t_{k}^{i}\right), \boldsymbol{x}_{-i}\left(t_{k}^{i}\right), \boldsymbol{u}_{i}^{*}\left(t_{k}^{i}\right), \boldsymbol{d}_{i}^{*}\left(t_{k}^{i}\right)\right)=\sum_{s=0}^{N-1} L_{i}$ $\left(x_{i}^{*}\left(s \mid t_{k}^{i}\right), x_{-i}\left(s \mid t_{k}^{i}\right), u_{i}^{*}\left(s \mid t_{k}^{i}\right), d_{i}^{*}\left(s \mid t_{k}^{i}\right)\right)+F_{i}\left(x_{i}^{*}\left(N \mid t_{k}^{i}\right)\right)$.

Proof: The proof of Lemma 4 can follow the idea in [24], so it is omitted here.

Theorem 1: Suppose Assumptions 1, 3 hold, and Problem $\mathcal{P}_{i}$ is feasible at the initial time $t_{0}^{i}$. 1) Then, by application of Algorithm 1, Problem $\mathcal{P}_{i}$ is recursively feasible at any sampling time $t_{k}^{i}, k \in \mathbb{I}_{[1,+\infty)}$, if the condition in Lemma 2 holds. 2) Furthermore, the closed-loop uncertain system with the self-triggered min-max DMPC strategy is ISpS at triggering time instants.

Proof: Feasibility: For agent $i, i \in \mathcal{M}$, by assumption, there is a feasible solution for Problem $\mathcal{P}_{i}$ at $t_{k}^{i}$ and the recursive feasibility for all subsequent triggering instants is proven by induction. The optimal control input sequence obtained at $t_{k}^{i}$ is $\boldsymbol{u}_{i}^{*}\left(t_{k}^{i}\right):=\left(u_{i}^{*}\left(0 \mid t_{k}^{i}\right), \ldots, u_{i}^{*}\left(H^{i *}-\right.\right.$ $\left.\left.1 \mid t_{k}^{i}\right), \mu_{i, \ell}^{*}\left(H^{i *} \mid t_{k}^{i}\right), \ldots, \mu_{i, 1}^{*}\left(N-1 \mid t_{k}^{i}\right)\right)$. The first $H^{i *}$ openloop control actions are implemented to the system in (2). At time $t_{k+1}^{i}$, a candidate control input sequence $\tilde{\boldsymbol{u}}_{i}\left(t_{k+1}^{i}\right)=$ $\tilde{u}_{i}\left(\cdot \mid t_{k+1}^{i}\right)$ can be constructed as

$$
\tilde{u}_{i}\left(s \mid t_{k+1}^{i}\right)= \begin{cases}\mu_{i, \ell}^{*}\left(H^{i *}+s \mid t_{k}^{i}\right), & s \in \mathbb{I}_{\left[0, N-H^{i *}\right)}, \\ \kappa_{i}\left(\tilde{x}_{i}\left(s \mid t_{k+1}^{i}\right)\right), & s \in \mathbb{I}_{\left[N-H^{i *}, N\right)} .\end{cases}
$$

Then the corresponding system states become $\tilde{x}_{i}(s+$ $\left.1 \mid t_{k+1}^{i}\right)=f_{i}\left(\tilde{x}_{i}\left(s \mid t_{k+1}^{i}\right), \tilde{u}_{i}\left(s \mid t_{k+1}^{i}\right), d_{i}^{*}\left(s+H^{i *} \mid t_{k}^{i}\right)\right)$, where $\tilde{x}_{i}\left(0 \mid t_{k+1}^{i}\right)=x_{i}\left(t_{k+1}^{i}\right)$. From Assumption 3 and the optimal feedback control input calculated at the previous time instant $t_{k}^{i}$, it follows that $\tilde{u}_{i}\left(s \mid t_{k+1}^{i}\right) \in \mathbb{U}_{i}, s \in \mathbb{I}_{[0, N)}$, the control input constraint (5b) is satisfied. For $s \in \mathbb{I}_{\left[0, N-H^{i *}\right]}$, we have the candidate state $\tilde{x}_{i}\left(s \mid t_{k+1}^{i}\right) \in \mathbb{X}_{i}^{N-H^{i *}-s}\left(\Omega_{i}\right) \subset \mathbb{X}_{i}$; then, for $s \in \mathbb{I}_{\left(N-H^{i *}, N\right]}$, under the terminal controller $\kappa_{i}\left(\tilde{x}_{i}\left(s \mid t_{k+1}^{i}\right)\right)$ given in Assumption 3 , the system state always belongs to the robust invariant set $\Omega_{i}$. Thus, the system state constraint $(5 \mathrm{c})$ is fulfilled. From Lemma 2, the feasibility of the constraint (5d) is guaranteed. The recursive feasibility of the proposed algorithm is established.

Stability: To prove the closed-loop stability, we need to show that the optimal value function $V_{i, N}^{H^{i *}}$ is an ISpS Lyapunov function at triggering time instants. Since the stage cost function $L_{i}$ for agent $i, i \in \mathcal{M}$ is quadratic and matrices $Q_{i}, Q_{i j}$ are positive definite, it can be derived that $L_{i} \geq \alpha_{L}\left(\left\|x_{i}\left(t_{k}^{i}\right)\right\|\right)$, where $\alpha_{L}\left(\left\|x_{i}\left(t_{k}^{i}\right)\right\|\right)=\underline{\lambda}\left(Q_{i}\right)\left\|x_{i}\left(t_{k}^{i}\right)\right\|^{2}$ is a $\mathcal{K}_{\infty}$ function. Then we obtain $V_{i, N}^{H^{i *}} \geq \alpha_{L}\left(\left\|x_{i}\left(t_{k}^{i}\right)\right\|\right)$. The similar way to establish the upper bound of $V_{i, N}^{H^{i *}}$ in [19] is adopted here. Define a set $B_{i, r}=\left\{x_{i} \in \mathbb{R}^{n}\right.$ | $\left.\left\|x_{i}\right\| \leq r_{i}\right\} \subseteq \Omega_{i}$. Due to the compactness of $\mathbb{X}_{i}$ and $\mathbb{U}_{i}$, the optimal value of the min-max DMPC cost function is upper bounded, i.e., $V_{i, N}^{H^{i *}}\left(x_{i}\left(t_{k}^{i}\right), \boldsymbol{x}_{-i}\left(t_{k}^{i}\right)\right) \leq \bar{V}_{i, N}$. If $x_{i}\left(t_{k}^{i}\right) \in \Omega_{i}$, by Assumption 3 and Lemma 3 , one can have

$$
\begin{aligned}
& F_{i}\left(x_{i}\left(s+1 \mid t_{k}^{i}\right)\right)-F_{i}\left(x_{i}\left(s \mid t_{k}^{i}\right)\right) \\
\leq & -L_{i}\left(x_{i}\left(s \mid t_{k}^{i}\right), x_{-i}\left(s \mid t_{k}^{i}\right), \kappa_{i}\left(x_{i}\left(s \mid t_{k}^{i}\right)\right), d_{i}\left(s \mid t_{k}^{i}\right)\right)+\epsilon_{i} .
\end{aligned}
$$


By summing up (10) from $s=0$ to $N$, we get

$$
\begin{aligned}
& \sum_{s=0}^{N-1} L_{i}\left(x_{i}\left(s \mid t_{k}^{i}\right), x_{-i}\left(s \mid t_{k}^{i}\right), \kappa_{i}\left(x_{i}\left(s \mid t_{k}^{i}\right)\right), d_{i}\left(s \mid t_{k}^{i}\right)\right) \\
& +F_{i}\left(x_{i}\left(N \mid t_{k}^{i}\right)\right) \\
\leq & F_{i}\left(x_{i}\left(t_{k}^{i}\right)\right)+N \epsilon_{i},
\end{aligned}
$$

where $F_{i}\left(x_{i}\left(t_{k}^{i}\right)\right)=F_{i}\left(x_{i}\left(0 \mid t_{k}^{i}\right)\right)$.

If the distributed triggering condition (7) is satisfied, i.e., $V_{i, N}^{H^{i *}}\left(x_{i}\left(t_{k}^{i}\right), \boldsymbol{x}_{-i}\left(t_{k}^{i}\right)\right) \leq V_{i, N}^{1}\left(x_{i}\left(t_{k}^{i}\right), \boldsymbol{x}_{-i}\left(t_{k}^{i}\right)\right)$. In the view of the definition of the terminal cost function, we have $F_{i}\left(x_{i}\left(t_{k}^{i}\right)\right) \leq \bar{\alpha}_{F}\left(\left\|x_{i}\left(t_{k}^{i}\right)\right\|\right)$, where $\bar{\alpha}_{F}\left(\left\|x_{i}\left(t_{k}^{i}\right)\right\|\right)=$ $\bar{\lambda}\left(P_{i}\right)\left\|x_{i}\left(t_{k}^{i}\right)\right\|^{2}$ is a $\mathcal{K}_{\infty}$ function. Then,

$$
\begin{aligned}
V_{i, N}^{H^{i *}}\left(x_{i}\left(t_{k}^{i}\right), \boldsymbol{x}_{-i}\left(t_{k}^{i}\right)\right) & \leq V_{i, N}^{1}\left(x_{i}\left(t_{k}^{i}\right), \boldsymbol{x}_{-i}\left(t_{k}^{i}\right)\right) \\
& \leq V_{i, N}\left(x_{i}\left(t_{k}^{i}\right), \boldsymbol{x}_{-i}\left(t_{k}^{i}\right)\right) \\
& \leq \bar{\alpha}_{F}\left(\left\|x_{i}\left(t_{k}^{i}\right)\right\|\right)+N \epsilon_{i} .
\end{aligned}
$$

The second inequality follows from the fact in Lemma 4 . If $x_{i}\left(t_{k}^{i}\right) \in \mathbb{X}_{i}^{N}\left(\Omega_{i}\right) \backslash \Omega_{i}$, it implies that $\bar{\alpha}_{F}\left(\left\|x_{i}\left(t_{k}^{i}\right)\right\|\right) \geq \bar{\alpha}_{F}\left(r_{i}\right)$, where $\mathbb{X}_{i}^{N}\left(\Omega_{i}\right)$ is the $N$-step robustly stabilizable set of agent $i$. And thus

$$
\begin{aligned}
V_{i, N}^{H^{i *}}\left(x_{i}\left(t_{k}^{i}\right), \boldsymbol{x}_{-i}\left(t_{k}^{i}\right)\right) & \leq \bar{V}_{i, N} \frac{\bar{\alpha}_{F}\left(\left\|x_{i}\left(t_{k}^{i}\right)\right\|\right)}{\bar{\alpha}_{F}\left(r_{i}\right)} \\
& \leq \theta_{i} \bar{\alpha}_{F}\left(\left\|x_{i}\left(t_{k}^{i}\right)\right\|\right)+N \epsilon_{i},
\end{aligned}
$$

where $\theta_{i}=\max \left\{1, \frac{\bar{V}_{i, N}}{\bar{\alpha}_{F}\left(r_{i}\right)}\right\}$.

At time $t_{k}^{i}$, the sequence of optimal control policies $\boldsymbol{u}_{i}^{*}\left(t_{k}^{i}\right)$ for Problem $\mathcal{P}_{i}$ can steer $x_{i}\left(t_{k}^{i}\right)$ of agent $i, i \in \mathcal{M}$ to the terminal set $\Omega_{i}$ in $N$ steps under the disturbance sequence $\boldsymbol{d}_{i}^{*}\left(t_{k}^{i}\right)=\left(d_{i}^{*}\left(0 \mid t_{k}^{i}\right), \ldots, d_{i}^{*}\left(N-1 \mid t_{k}^{i}\right)\right)$. If the control actions $\boldsymbol{u}_{i}^{\mathrm{mpc}}\left(t_{k}^{i}\right)$ and the actual disturbance $\boldsymbol{d}_{i}^{a}\left(t_{k}^{i}\right)$ are applied, then agent $i$ evolves to $x_{i}\left(t_{k+1}^{i}\right)$, where $\boldsymbol{d}_{i}^{a}\left(t_{k}^{i}\right)=\left(d_{i}\left(t_{k}^{i}\right), d_{i}\left(t_{k}^{i}+\right.\right.$ $\left.1), \ldots, d_{i}\left(t_{k}^{i}+H^{i *}-1\right)\right)$. For agent $j, j \in \mathcal{N}_{i}$, the broadcast state evolves to $x_{j}^{*}\left(H^{i *} \mid t_{k}^{i}\right)$ with the control actions $u_{j}^{*}\left(s \mid t_{k}^{i}\right)$ and the disturbance input $d_{j}^{*}\left(s \mid t_{k}^{i}\right), s \in \mathbb{I}_{\left[0, H^{i *}\right)}$.

Then, it is easy to have

$$
\begin{aligned}
& J_{i, N-H^{i *}}\left(x_{i}\left(t_{k+1}^{i}\right), \boldsymbol{x}_{-i}\left(t_{k+1}^{i}\right), \boldsymbol{\mu}_{i}^{*}\left(t_{k}^{i}\right), \boldsymbol{d}_{i}^{\prime}\left(t_{k}^{i}\right)\right) \\
= & J_{i, N}^{H^{i *}}\left(x_{i}\left(t_{k}^{i}\right), \boldsymbol{x}_{-i}\left(t_{k}^{i}\right), \boldsymbol{u}_{i}^{*}\left(t_{k}^{i}\right), \boldsymbol{d}_{i}^{\prime \prime}\left(t_{k}^{i}\right)\right) \\
& -\frac{1}{\hbar_{i}} \sum_{s=0}^{H^{i *}-1} L_{i}\left(x_{i}\left(s \mid t_{k}^{i}\right), x_{-i}\left(s \mid t_{k}^{i}\right), u_{i}^{*}\left(s \mid t_{k}^{i}\right), \boldsymbol{d}_{i}^{a}\left(t_{k}^{i}\right)\right),
\end{aligned}
$$

where $\boldsymbol{\mu}_{i}^{*}\left(t_{k}^{i}\right)=\left(\mu_{i, N-H^{i *}}^{*}\left(H^{i *} \mid t_{k}^{i}\right), \ldots, \mu_{i, 1}^{*}\left(N-1 \mid t_{k}^{i}\right)\right)$, $\boldsymbol{d}_{i}^{\prime}\left(t_{k}^{i}\right)=\left(d_{i}^{*}\left(0 \mid t_{k+1}^{i}\right), \ldots, d_{i}^{*}\left(N-H^{i *}-1 \mid t_{k+1}^{i}\right)\right)$ and $\boldsymbol{d}_{i}^{\prime \prime}\left(t_{k}\right)=\left(d_{i}\left(t_{k}^{i}\right), \ldots, d_{i}\left(t_{k}^{i}+H^{i *}-\right.\right.$ $\left.1), d_{i}^{*}\left(0 \mid t_{k+1}^{i}\right), \ldots, d_{i}^{*}\left(N-H^{i *}-1 \mid t_{k+1}^{i}\right)\right)$. Then, from the above equality, we get

$$
\begin{aligned}
& J_{i, N-H^{i *}}\left(x_{i}\left(t_{k+1}^{i}\right), \boldsymbol{x}_{-i}\left(t_{k+1}^{i}\right), \boldsymbol{\mu}_{i}^{*}\left(t_{k}^{i}\right), \boldsymbol{d}_{i}^{\prime}\left(t_{k}^{i}\right)\right) \\
\leq & J_{i, N}^{H^{i *}}\left(x_{i}\left(t_{k}^{i}\right), \boldsymbol{x}_{-i}\left(t_{k}^{i}\right), \boldsymbol{u}_{i}^{*}\left(t_{k}^{i}\right), \boldsymbol{d}_{i}^{\prime \prime}\left(t_{k}^{i}\right)\right) \\
& -H^{i *} / \hbar_{i} \alpha_{L}\left(\left\|x_{i}\left(t_{k}^{i}\right)\right\|\right)
\end{aligned}
$$

Further, the system state $x_{i}\left(t_{k+1}^{i}\right)$ can be steered into the terminal set $\Omega_{i}$ in $N-H^{i *}$ steps by the candidate control input $\tilde{\boldsymbol{u}}_{i}\left(t_{k+1}^{i}\right)$. Then, by the inequality (13), and Assumption 3

$$
\begin{aligned}
& J_{i, N}\left(x_{i}\left(t_{k+1}^{i}\right), \boldsymbol{x}_{-i}\left(t_{k+1}^{i}\right), \tilde{\boldsymbol{u}}_{i}\left(t_{k+1}^{i}\right), \boldsymbol{d}_{i}^{*}\left(t_{k+1}^{i}\right)\right) \\
\leq & J_{i, N}^{H^{i *}}\left(x_{i}\left(t_{k}^{i}\right), \boldsymbol{x}_{-i}\left(t_{k}^{i}\right), \boldsymbol{u}_{i}^{*}\left(t_{k}^{i}\right), \boldsymbol{d}_{i}^{\prime \prime}\left(t_{k}^{i}\right)\right) \\
& -H^{i *} / \hbar_{i} \alpha_{L}\left(\left\|x_{i}\left(t_{k}^{i}\right)\right\|\right)+H^{i *} \epsilon_{i},
\end{aligned}
$$

where $\boldsymbol{d}_{i}^{*}\left(t_{k+1}^{i}\right)=\left(d_{i}^{*}\left(0 \mid t_{k+1}^{i}\right), \ldots, d_{i}^{*}\left(N-1 \mid t_{k+1}^{i}\right)\right)$.

Consider the time-varying communication delays in Case 2, i.e., $t_{k+1}^{i}-t_{k+1}^{i j}<\tau_{k+1}^{i j} \leq \bar{\tau}$, the predicted states of neighbors transmitted at the previous triggering time instant $\boldsymbol{x}_{-i}\left(t_{k+1}^{i}\right)=$ $\left(x_{-i}\left(H^{i *} \mid t_{k}^{i}\right), \ldots, x_{-i}\left(H^{i *}+N-1 \mid t_{k}^{i}\right)\right)$ will be used. Based on the triggering condition and Lemma 4, we obtain

$$
\begin{aligned}
& V_{i, N}^{H^{i *}\left(t_{k+1}^{i}\right)}\left(x_{i}\left(t_{k+1}^{i}\right), \boldsymbol{x}_{-i}\left(t_{k+1}^{i}\right)\right) \\
\text { ?] } & V_{i, N}^{1}\left(x_{i}\left(t_{k+1}^{i}\right), \boldsymbol{x}_{-i}\left(t_{k+1}^{i}\right)\right) \\
\leq & V_{i, N}\left(x_{i}\left(t_{k+1}^{i}\right), \boldsymbol{x}_{-i}\left(t_{k+1}^{i}\right)\right) \\
= & J_{i, N}\left(x_{i}\left(t_{k+1}^{i}\right), \boldsymbol{x}_{-i}\left(t_{k+1}^{i}\right), \boldsymbol{u}_{i}^{*}\left(t_{k+1}^{i}\right), \boldsymbol{d}_{i}^{*}\left(t_{k+1}^{i}\right)\right) \\
\leq & J_{i, N}\left(x_{i}\left(t_{k+1}^{i}\right), \boldsymbol{x}_{-i}\left(t_{k+1}^{i}\right), \tilde{\boldsymbol{u}}_{i}\left(t_{k+1}^{i}\right), \boldsymbol{d}_{i}^{*}\left(t_{k+1}^{i}\right)\right) \\
\underline{14} & J_{i, N}^{H^{i *}}\left(x_{i}\left(t_{k}^{i}\right), \boldsymbol{x}_{-i}\left(t_{k}^{i}\right), \boldsymbol{u}_{i}^{*}\left(t_{k}^{i}\right), \boldsymbol{d}_{i}^{\prime \prime}\left(t_{k}^{i}\right)\right) \\
& -H^{i *} / \hbar_{i} \alpha_{L}\left(\left\|x_{i}\left(t_{k}^{i}\right)\right\|\right)+H^{i *} \epsilon_{i} \\
\leq & V_{i, N}^{H^{i *}}\left(x_{i}\left(t_{k}^{i}\right), \boldsymbol{x}_{-i}\left(t_{k}^{i}\right)\right)-H^{i *} / \hbar_{i} \alpha_{L}\left(\left\|x_{i}\left(t_{k}^{i}\right)\right\|\right) \\
& +H^{i *} \epsilon_{i} .
\end{aligned}
$$

The last inequality is derived from the fact that $\boldsymbol{d}_{i}^{\prime \prime}\left(t_{k}^{i}\right)$ is not the optimal solution of Problem $\mathcal{P}_{i}$.

Consider the time-varying communication delays in Case 1, i.e., $\tau_{k+1}^{i j} \leq t_{k+1}^{i}-t_{k+1}^{i j}$, the newest predicted states of neighbors $\boldsymbol{x}_{-i}^{\prime}\left(t_{k+1}^{i}\right)=\left(x_{-i}\left(0 \mid t_{k+1}^{i}\right), \ldots, x_{-i}\left(N-1 \mid t_{k+1}^{i}\right)\right)$ will be received and used by agent $i$. Because of the triangle inequality and the consistency constraint (5d), we have

$$
\begin{aligned}
& \quad\left\|x_{i}\left(s \mid t_{k+1}^{i}\right)-x_{j}\left(s \mid t_{k+1}^{i}\right)\right\| \\
& \leq\left\|x_{i}\left(s \mid t_{k+1}^{i}\right)-x_{j}^{b}\left(H^{i *}+s \mid t_{k}^{i}\right)\right\| \\
& \quad+\left\|x_{j}\left(s \mid t_{k+1}^{i}\right)-x_{j}^{b}\left(H^{i *}+s \mid t_{k}^{i}\right)\right\| \\
& \stackrel{\text { 5d| }}{\leq}\left\|x_{i}\left(s \mid t_{k+1}^{i}\right)-x_{j}^{b}\left(H^{i *}+s \mid t_{k}^{i}\right)\right\|+\Delta_{j} .
\end{aligned}
$$

Since $x_{i}\left(\cdot \mid t_{k}^{i}\right) \in \mathbb{X}_{i}$, there exists a constant $\Lambda_{i}>0$ such that $\left\|x_{i}\left(s \mid t_{k}^{i}\right)\right\| \leq \Lambda_{i}, k \in \mathbb{I}$. Then it can be obtained that

$$
\begin{aligned}
& \quad\left\|x_{i}\left(s \mid t_{k+1}^{i}\right)-x_{j}\left(s \mid t_{k+1}^{i}\right)\right\|_{Q_{i j}}^{2} \\
& \leq\left\|x_{i}\left(s \mid t_{k+1}^{i}\right)-x_{j}^{b}\left(H^{i *}+s \mid t_{k}^{i}\right)\right\|_{Q_{i j}}^{2} \\
& \quad+2 \bar{\lambda}\left(Q_{i j}\right)\left\|x_{i}\left(s \mid t_{k+1}^{i}\right)-x_{j}^{b}\left(H^{i *}+s \mid t_{k}^{i}\right)\right\| \\
& \quad \cdot\left\|x_{j}\left(s \mid t_{k+1}^{i}\right)-x_{j}^{b}\left(H^{i *}+s \mid t_{k}^{i}\right)\right\| \\
& \quad+\left\|x_{j}\left(s \mid t_{k+1}^{i}\right)-x_{j}^{b}\left(H^{i *}+s \mid t_{k}^{i}\right)\right\|_{Q_{i j}}^{2} \\
& \text { 卢) } \\
& \leq x_{i}\left(s \mid t_{k+1}^{i}\right)-x_{j}^{b}\left(H^{i *}+s \mid t_{k}^{i}\right) \|_{Q_{i j}}^{2} \\
& \quad+4 \bar{\lambda}\left(Q_{i j}\right) \Lambda_{i} \Delta_{j}+\bar{\lambda}\left(Q_{i j}\right) \Delta_{j}^{2} .
\end{aligned}
$$


From the triggering condition and Lemma 3, we obtain that

$$
\begin{aligned}
& V_{i, N}^{H^{i *}\left(t_{k+1}^{i}\right)}\left(x_{i}\left(t_{k+1}^{i}\right), \boldsymbol{x}_{-i}^{\prime}\left(t_{k+1}^{i}\right)\right) \\
& \text { 곤 } V_{i, N}^{1}\left(x_{i}\left(t_{k+1}^{i}\right), \boldsymbol{x}_{-i}^{\prime}\left(t_{k+1}^{i}\right)\right) \\
& \leq V_{i, N}\left(x_{i}\left(t_{k+1}^{i}\right), \boldsymbol{x}_{-i}^{\prime}\left(t_{k+1}^{i}\right)\right) \\
& =J_{i, N}\left(x_{i}\left(t_{k+1}^{i}\right), \boldsymbol{x}_{-i}^{\prime}\left(t_{k+1}^{i}\right), \boldsymbol{u}_{i}^{*}\left(t_{k+1}^{i}\right), \boldsymbol{d}_{i}^{*}\left(t_{k+1}^{i}\right)\right) \\
& \leq J_{i, N}\left(x_{i}\left(t_{k+1}^{i}\right), \boldsymbol{x}_{-i}^{\prime}\left(t_{k+1}^{i}\right), \tilde{\boldsymbol{u}}_{i}\left(t_{k+1}^{i}\right), \boldsymbol{d}_{i}^{*}\left(t_{k+1}^{i}\right)\right) \\
& \stackrel{17}{\leq} J_{i, N}\left(x_{i}\left(t_{k+1}^{i}\right), \boldsymbol{x}_{-i}\left(t_{k+1}^{i}\right), \tilde{\boldsymbol{u}}_{i}\left(t_{k+1}^{i}\right), \boldsymbol{d}_{i}^{*}\left(t_{k+1}^{i}\right)\right) \\
& +\left(N-H^{i *}\right) \bar{\lambda}\left(Q_{i j}\right) \sum_{j \in \mathcal{N}_{i}}\left(4 \Lambda_{i} \Delta_{j}+\Delta_{j}^{2}\right) \\
& \leq J_{i, N}^{H^{i *}}\left(x_{i}\left(t_{k}^{i}\right), \boldsymbol{x}_{-i}\left(t_{k}^{i}\right), \boldsymbol{u}_{i}^{*}\left(t_{k}^{i}\right), \boldsymbol{d}_{i}^{\prime \prime}\left(t_{k}^{i}\right)\right) \\
& -H^{i *} / \hbar_{i} \alpha_{L}\left(\left\|x_{i}\left(t_{k}^{i}\right)\right\|\right)+\Xi_{i} \\
& \leq V_{i, N}^{H^{i *}}\left(x_{i}\left(t_{k}^{i}\right), \boldsymbol{x}_{-i}\left(t_{k}^{i}\right)\right)-H^{i *} / \hbar_{i} \alpha_{L}\left(\left\|x_{i}\left(t_{k}^{i}\right)\right\|\right)+\Xi_{i},
\end{aligned}
$$

where $\Xi_{i}=\left(N-H^{i *}\right) \bar{\lambda}\left(Q_{i j}\right) \sum_{j \in \mathcal{N}_{i}}\left(4 \Lambda_{i} \Delta_{j}+\Delta_{j}^{2}\right)+H^{i *} \epsilon_{i}$. By now, we have shown that $V_{i, N}^{H^{i *}}\left(x_{i}\left(t_{k}^{i}\right), \boldsymbol{x}_{-i}\left(t_{k}^{i}\right)\right)$ is an ISpS Lyapunov function. Based on Lemma 1, it can be concluded that the closed-loop system in (8) is ISpS with respect to $\Omega_{i}$ at triggering instants. This concludes the proof.

\section{NUMERICAL EXAMPLE}

Three group of simulations are conducted i.e., periodic min-max DMPC (P-DMPC), self-triggered min-max DMPC without delays (ST-DMPC) and self-triggered min-max DMPC with delays (ST-DMPC-D). Consider MAS with five agents adopted in [24], and agent $i, i \in \mathcal{M}$ is characterized by

$$
\begin{aligned}
& x_{i, 1}^{+}=x_{i, 1}+T\left(x_{i, 2}\right), \\
& x_{i, 2}^{+}=x_{i, 2}-\frac{T}{m_{i}}\left(k_{i}^{\prime} e^{-x_{i, 1}} x_{i, 1}+h_{i}^{\prime} x_{i, 2}-u_{i}+v_{i} x_{i, 2}-w_{i}\right),
\end{aligned}
$$

in which $m_{i}=1 \mathrm{~kg}, k_{i}^{\prime}=0.33 \mathrm{~N} / \mathrm{m}, h_{i}^{\prime}=1.1 \mathrm{Ns} / \mathrm{m}$ and $T=0.3 \mathrm{~s}$. The uncertainties are bounded by $-0.1 \leq v_{i} \leq 0.1$, $-0.15 \leq w_{i} \leq 0.15$. The control input and state constraints are given by $-4 \mathrm{~N} \leq u_{i} \leq 4 \mathrm{~N},-1.95 \mathrm{~m} \leq x_{i, 1} \leq 1.95 \mathrm{~m}$. The Lipschitz constants can be calculated as $\nu=1.23$ and $\xi=0.42$ (see Lemma 3.2 in [22]). The delay $\tau \in \mathbb{I}_{[1, \bar{\tau}]}$ is randomly generated, with $\bar{\tau}=3$. Other parameters are designed as follows: $Q_{i}=\operatorname{diag}(0.6,0.6), Q_{i j}=\operatorname{diag}(0.5,0.5)$, $P_{i}=[8.05,2.90 ; 2.90,3.48], R_{i}=1, N=5, \bar{H}=4$, $\hbar_{i}=1.1, \Delta_{i}=3.58$ and $\rho_{i}=\sqrt{6.0}$. The terminal control law is $\kappa_{i}\left(x_{i}\right)=[-0.87,-1.04] x_{i}$. The feedback control policy $\mu_{i}\left(x_{i}\right)=a \kappa_{i}\left(x_{i}\right)+b\left\|x_{i}\right\|^{2}+c$, where $a, b, c \in \mathbb{R}$ are decision variables. The parametric uncertainties $v_{i}(t)$ and external disturbances $w_{i}(t)$ are $0.1 \sin (t / 4 \pi)$ and $0.15 \cos (t / 3 \pi)$, respectively. $\mathcal{G}$ is described by $\mathcal{N}_{1}=\{2\}, \mathcal{N}_{2}=\{1,5\}, \mathcal{N}_{3}=\{2,4\}$, $\mathcal{N}_{4}=\{3\}, \mathcal{N}_{5}=\{2\}$. The initial states are given as follows: $x_{1}=[1.5,0.7]^{\mathrm{T}}, x_{2}=[-0.5,-1.1]^{\mathrm{T}}, x_{3}=[-2.0,0.5]^{\mathrm{T}}$, $x_{4}=[0.7,-1.0]^{\mathrm{T}}, x_{5}=[1.95,0]^{\mathrm{T}}$.

Fig. 2 depicts five agents' state trajectories and triggering instants without communication delays under the P-DMPC method and ST-DMPC. The state trajectories of five agents with time-varying communication delays are shown in Fig. 3.

Let $\bar{T}:=\sum_{i \in \mathcal{M}} T_{\text {sim }} / M s_{i}$ denote the average sampling time, where $s_{i}$ is total triggering times of agent $i$ during

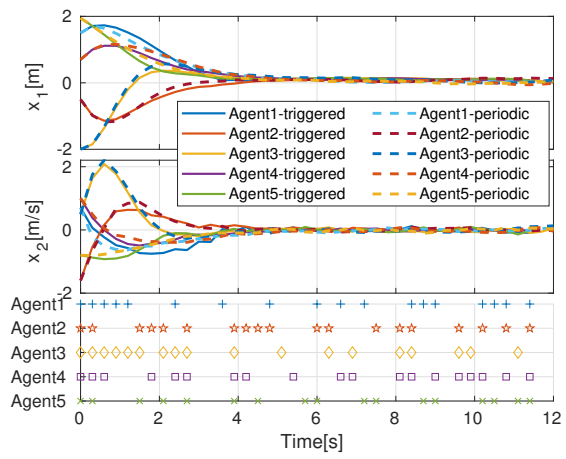

Fig. 2: State trajectories and triggering instants of P-DMPC and ST-DMPC. Top: The displacements. Middle: The velocities. Bottom: The triggering instants.

the simulation time $T_{\text {sim }}$. The control performance index is defined by $\bar{J}:=\sum_{i \in \mathcal{M}} J_{i} / M, J_{i}=\sum_{t=0}^{T_{\text {sim }}}\left\|x_{i}(t)\right\|_{Q_{i}}^{2}+$ $\sum_{j \in \mathcal{N}_{i}}\left\|x_{i}(t)-x_{j}(t)\right\|_{Q_{i j}}^{2}+\left\|u_{i}(t)\right\|_{R_{i}}^{2}$. From Table 1, it can be seen that the communication load of MAS is significantly reduced by using ST-DMPC(-D) while achieving comparable control performance of P-DMPC.

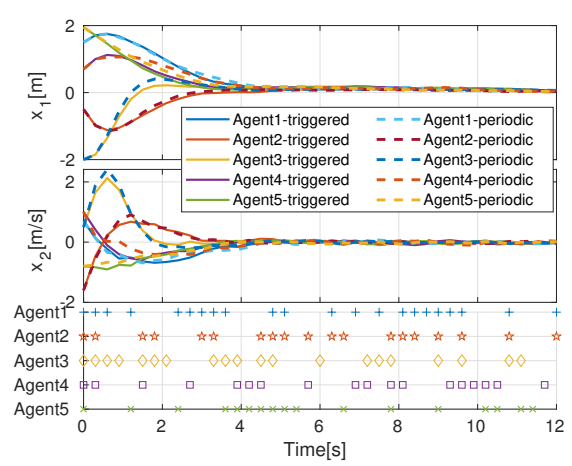

Fig. 3: State trajectories and triggering instants of P-DMPC and ST-DMPC-D. Top: The displacements. Middle: The velocities. Bottom: The triggering instants.

TABLE I: Performance comparison.

\begin{tabular}{lcc}
\hline Method & Average sampling time & Control performance index \\
\hline P-DMPC & 0.3000 & 51.3016 \\
ST-DMPC & 0.6303 & 52.2235 \\
ST-DMPC-D & 0.6105 & 51.4515 \\
\hline
\end{tabular}

\section{CONCLUSION}

This paper proposed a self-triggered min-max DMPC for nonlinear perturbed MAS with communication delays. A novel consistency constraint was designed to force the deviation between the newest and previously predicted states to lie in a prescribed region. The proposed method significantly reduced the communication load with slightly affected closed-loop performance than the periodic DMPC method. As mentioned above, the control inputs were obtained by solving the minmax optimal control problem at triggering instants, which, 
however, was the main limitation of the proposed method due to the lack of efficient solvers to the nonlinear min-max optimization problem. Future work will focus on reducing the computational burden of the min-max DMPC.

\section{REFERENCES}

[1] P. Liu, A. Kurt, and U. Ozguner, "Distributed model predictive control for cooperative and flexible vehicle platooning," IEEE Trans. Control Syst. Technol., no. 99, pp. 1-14, 2018.

[2] L. Guo, B. Yang, J. Ye, H. Chen, F. Li, W. Song, L. Du, and L. Guan, "Systematic assessment of cyber-physical security of energy management system for connected and automated electric vehicles," IEEE Trans. Ind. Informat., vol. 17, no. 5, pp. 3335-3347, 2021.

[3] C. Li, X. Yu, W. Yu, T. Huang, and Z.-W. Liu, "Distributed eventtriggered scheme for economic dispatch in smart grids," IEEE Trans. Ind. Informat., vol. 12, no. 5, pp. 1775-1785, 2015.

[4] M. S. Rahman, M. A. Mahmud, A. M. T. Oo, and H. R. Pota, "Multiagent approach for enhancing security of protection schemes in cyberphysical energy systems," IEEE Trans. Ind. Informat., vol. 13, no. 2, pp. 436-447, 2017.

[5] Z. Feng, C. Sun, and G. Hu, "Robust connectivity preserving rendezvous of multirobot systems under unknown dynamics and disturbances," IEEE Trans. Control. Netw. Syst., vol. 4, no. 4, pp. 725-735, 2016.

[6] D. V. Dimarogonas, E. Frazzoli, and K. H. Johansson, "Distributed event-triggered control for multi-agent systems," IEEE Trans. Autom. Control, vol. 57, no. 5, pp. 1291-1297, 2011.

[7] L. Wang and J. Dong, "Adaptive fuzzy consensus tracking control for uncertain fractional-order multi-agent systems with event-triggered input," IEEE Trans. Fuzzy Syst., pp. 1-1, 2020, doi 10.1109/TFUZZ.2020.3037957

[8] Y.-W. Wang, Y. Lei, T. Bian, and Z.-H. Guan, "Distributed control of nonlinear multiagent systems with unknown and nonidentical control directions via event-triggered communication," IEEE Trans. Cybern., vol. 50, no. 5, pp. 1820-1832, 2019.

[9] E. Tian and C. Peng, "Memory-based event-triggering $H_{\infty}$ load frequency control for power systems under deception attacks," IEEE Trans. Cybern., vol. 50, no. 11, pp. 4610-4618, 2020.

[10] D. Groß and O. Stursberg, "A cooperative distributed MPC algorithm with event-based communication and parallel optimization," IEEE Trans. Control. Netw. Syst., vol. 3, no. 3, pp. 275-285, 2015.

[11] Y. Zou, X. Su, S. Li, Y. Niu, and D. Li, "Event-triggered distributed predictive control for asynchronous coordination of multi-agent systems," Automatica, vol. 99, pp. 92-98, 2019.

[12] A. S. Kolarijani, S. C. Bregman, P. M. Esfahani, and T. Keviczky, "A decentralized event-based approach for robust model predictive control," IEEE Trans. Autom. Control, vol. 65, no. 8, pp. 3517-3529, 2020.

[13] X. Mi, Y. Zou, S. Li, and H. R. Karimi, "Self-triggered DMPC design for cooperative multi-agent systems," IEEE Trans. Ind. Electron., vol. 67, no. 1, pp. 512-520, 2020.

[14] J. Zhan, Z.-P. Jiang, Y. Wang, and X. Li, "Distributed model predictive consensus with self-triggered mechanism in general linear multiagent systems," IEEE Trans. Ind. Informat., vol. 15, no. 7, pp. 3987-3997, 2019.

[15] L. Fang and P. J. Antsaklis, "Information consensus of asynchronous discrete-time multi-agent systems," in Proc. of the American Control Conference (ACC'05), Portland, Oregon, 2005, pp. 1883-1888.

[16] G. P. Incremona, A. Ferrara, and L. Magni, "Asynchronous networked MPC with ISM for uncertain nonlinear systems," IEEE Trans. Autom. Control, vol. 62, no. 9, pp. 4305-4317, 2017.

[17] H. Li and Y. Shi, Robust Receding Horizon Control for Networked and Distributed Nonlinear Systems. New York, NY, USA: Springer, 2017.

[18] S. El-Ferik, B. A. Siddiqui, and F. L. Lewis, "Distributed nonlinear MPC of multi-agent systems with data compression and random delays," IEEE Trans. Autom. Control, vol. 61, no. 3, pp. 817-822, 2015.

[19] D. Limón, T. Alamo, F. Salas, and E. F. Camacho, "Input to state stability of min-max MPC controllers for nonlinear systems with bounded uncertainties," Automatica, vol. 42, no. 5, pp. 797-803, 2006.

[20] M. Lazar, D. M. De La Peña, W. Heemels, and T. Alamo, "On inputto-state stability of min-max nonlinear model predictive control," Syst. Control Lett., vol. 57, no. 1, pp. 39-48, 2008.

[21] W. B. Dunbar, "Distributed receding horizon control of dynamically coupled nonlinear systems," IEEE Trans. Autom. Control, vol. 52, no. 7, pp. 1249-1263, 2007.

[22] H. K. Khalil, Nonlinear Systems. Upper Saddle River, NJ, USA: Prentice-Hall, 2002.
[23] F. Xiao and L. Wang, "Asynchronous consensus in continuous-time multi-agent systems with switching topology and time-varying delays," IEEE Trans. Autom. Control, vol. 53, no. 8, pp. 1804-1816, 2008.

[24] C. Liu, H. Li, J. Gao, and D. Xu, "Robust self-triggered min-max model predictive control for discrete-time nonlinear systems," Automatica, vol. 89, pp. 333-339, 2018.

[25] F. D. Brunner, M. Heemels, and F. Allgöwer, "Robust self-triggered MPC for constrained linear systems: A tube-based approach," Automatica, vol. 72, pp. 73-83, 2016.

[26] W. B. Dunbar and R. M. Murray, "Distributed receding horizon control for multi-vehicle formation stabilization," Automatica, vol. 42, no. 4, pp. 549-558, 2006.

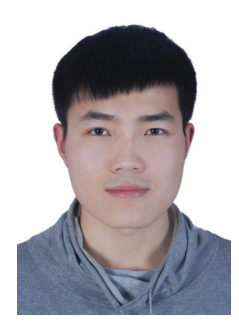

Henglai Wei (S'17) received his B.S. (Hons.) and M.S. degrees in control theory from Northwestern Polytechnical University, Xi' an, China, in 2014 and 2017 , respectively. He is currently working toward his Ph.D. degree in control theory with the University of Victoria, Canada. His current research interests include distributed model predictive control and optimization with applications on autonomous marine robots.

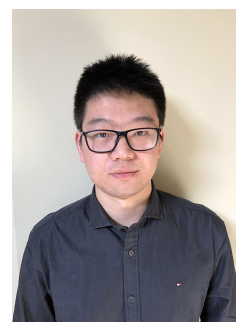

Kunwu Zhang (S'16) received the B.Eng. degree in electrical engineering and automation from Hubei University of Science and Technology, Xianning, China, in 2013, and the M.A.Sc. degree in mechanical engineering from the University of Victoria, Victoria, BC, Canada, in 2016, where he is currently working toward the Ph.D. degree in the Department of Mechanical Engineering. His current research interests include adaptive control, model predictive control, optimization and robotic systems.

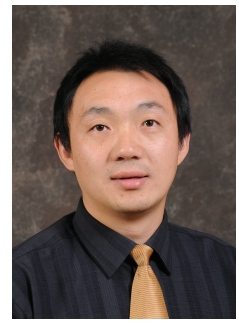

Yang Shi (SM'09-F'17) received the Ph.D. degree in electrical and computer engineering from the University of Alberta, Edmonton, AB, Canada, in 2005. From 2005 to 2009, he was an Assistant Professor and Associate Professor in the Department of Mechanical Engineering, University of Saskatchewan, Saskatoon, SK, Canada. In 2009, he joined the University of Victoria, and now he is a Professor in the Department of Mechanical Engineering, University of Victoria, Victoria, BC, Canada. His current research interests include networked and distributed systems, model predictive control (MPC), cyber-physical systems (CPS), robotics and mechatronics, control of autonomous systems (AUV and UAV), and energy system applications. Dr. Shi received the University of Saskatchewan Student Union Teaching Excellence Award in 2007, and the Faculty of Engineering Teaching Excellence Award in 2012 at the University of Victoria (UVic). He is the recipient of the JSPS Invitation Fellowship (short-term) in 2013, the UVic Craigdarroch Silver Medal for Excellence in Research in 2015, the 2017 IEEE Transactions on Fuzzy Systems Outstanding Paper Award, the Humboldt Research Fellowship for Experienced Researchers in 2018. Currently he serves as a member of the IEEE IES Administrative Committee, the Chair of IEEE IES Technical Committee on Industrial Cyber-Physical Systems, and Co-Editor-in-Chief for IEEE Transactions on Industrial Electronics. He also serves as Associate Editor for Automatica, IEEE Transactions on Control Systems Technology, IEEE/ASME Transactions on Mechatronics, IEEE Transactions on Cybernetics, etc. He is General Chair of the 2019 International Symposium on Industrial Electronics (ISIE) and the 2021 International Conference on Industrial Cyber-Physical Systems (ICPS). He is a Fellow of IEEE, ASME, CSME, and Engineering Institute of Canada (EIC), and a registered Professional Engineer in British Columbia, Canada. 